\title{
LA ESTRUCTURA DEL DISCURSO IG EN ESPAÑA: ELEMENTOS CLAVE PARA LA TRANSFORMACIÓN HACIA UN SISTEMA SOSTENIBLE.
}

\section{Omar V. GUADARRAMA-FUENTES ${ }^{*}$, José M. GIL ${ }^{1}$.}

${ }^{1}$ CREDA-UPC-IRTA. *omar.vicente.guadarrama@upc.edu

Desde la implementación del sistema de indicaciones geográfica europeo en 1996, la investigación académica para identificar, cuantificar y evaluar los alcances y oportunidades del sistema han crecido exponencialmente.

Existe una gran literatura, española e internacional, que documenta los beneficios económicos, medio ambientales y sociales, así como los casos de éxito de la implementación de las IG en diferentes latitudes. Sin embargo, ninguno de los trabajos se ha enfocado en analizar la percepción que tienen los actores involucrados. Información necesaria para construir las políticas publicas con una perspectiva sostenible. Para ello, en este trabajo a partir de la metodóloga Q, una herramienta mixta, se seleccionaron 44 afirmaciones procedente de literatura, las cuales se presentaron a una muestra de 35 personas en representación de consejos reguladores, administración púbica y académicos. Los resultados muestran la existencia de tres argumentos en torno al discurso IG: uno que toma la visión institucional, un segundo que destaca la parte tradicional y cultural del producto; y un tercero que incorpora una visión de la sostenibilidad, los perfiles se han asociado con las características de cada una de las personas que ha participado en el estudio para tener una mayor comprensión de los argumentos.

Palabras clave: Discurso IG, Metodología Q, sistema agroalimentario sostenible.

\section{Introducción}

Las indicaciones geográficas (IG) son un método de producción multifunción de la agroindustria que constituye una alternativa a la intensificación y la estandarización, se basa en el patrón dietético de una zona incorporando elementos que permiten el desarrollo regional respetando los recursos naturales y con un impacto social positivo. En el caso de España y Europa se emplean dos sellos distintivos de calidad asociados al origen, la Denominación de Origen Protegida (DOP) y la Indicación Geográfica Protegida (IGP) (Fierro \& Martín, 2009; Millán Vázquez De La Torre et al., 2014)

En el caso de España, las IG son percibidas como un sistema territorial vinculado al origen cuya calidad se sustenta en un trasfondo cultural que permite una transición de los sistemas productivos, integrando una visión holística que derive en un sistema agroalimentario sostenible mediante la reterritorialización de las zonas rurales y periurbanas (Esnouf et al., 2013; García Romana, 2017; Ramírez García, 2016). Sin embargo, los estudios realizados en materia de sostenibilidad de los sistemas IG en España son escasos, de ahí el interés del presente trabajo por mostrar los resultados de la aplicación de la metodología Q a un grupo de actores involucrados en el sistema IG, consejos reguladores (CR), autoridad publica e investigadores, donde se identificaron los principales argumentos que motivan la implementación del sistema IG y las características de quienes coinciden en cada grupo de opinión.

\section{Metodología}

La metodología Q diseñada por William Stephenson en 1935, nos permite estudia la subjetividad de los participantes por medio de un análisis factorial, el cual se aplica por medio de 5 fases (Brown, 1993; Roth Deubel \& Bernal Gamboa, 2014)..

La primera fase o Q-Sample, consiste en la recopilación de todas las ideologías, argumentos y puntos de vista en torno al tema de estudio basado en una revisión bibliográfica de 26 documentos publicados entre 1997 y 2018 que incluyen literatura científica revisada por pares y literatura gris, identificando 4 principales argumentos: económico, socio-económico, económico-ambiental y sostenible. La segunda fase consistió en seleccionar 44 afirmaciones representativas del Q-Sample. En la tercera fase se integró el P-Sample, integrado por 35 personas: 18 integrantes de consejos reguladores, 5 representantes 
de las comunidades autónomas y 12 investigadores. La cuarta fase consiste en la aplicación del Q-Sorting, es decir, se deben organizar las 44 afirmaciones por afinidad personal (de acuerdo, en desacuerdo), para distribuirlas en una cuadricula simétrica con un valor central de 0 y valores extremos de -5 . La última fase consiste en le análisis de los datos con el software libre PQMethod versión 2.35, aplicando una extracción de factores centroides bajo el criterio de Horst, ya que parece que responde mejor al enfoque filosófico subyacente de la metodología Q y los objetivos de este trabajo (Darton, 1980; Ramlo, 2016). Asimismo, se aplicó una rotación varimax para la obtención de los factores.

\section{Resultados}

Los resultados obtenidos del análisis factorial muestran tres factores con valores propios superiores a la unidad, los cuales asocian a 30 de los participantes a uno de los factores (Tabla 2), mientras que los 5 restantes no han podido asociarse a ningún factor. La correlación residual cuadra asciende a 0,019 y la varianza explicada acumulada es del $38 \%$.

Tabla 2. Participantes que integran cada factor.

\begin{tabular}{|c|c|c|c|}
\hline \multicolumn{4}{|c|}{ Participantes que integran el perfil 1} \\
\hline \begin{tabular}{|l}
$\begin{array}{l}\text { Código del } \\
\text { participante }\end{array}$ \\
\end{tabular} & \begin{tabular}{|c} 
Factor \\
1 \\
\end{tabular} & $\begin{array}{c}\text { Factor } \\
\mathbf{2}\end{array}$ & \begin{tabular}{|c} 
Facto \\
$\mathbf{3}$
\end{tabular} \\
\hline 1602 & $0.54 \mathrm{x}$ & 0.21 & -0.22 \\
\hline 1605 & $0.44 x$ & 0.26 & $\mid-0.11$ \\
\hline 1607 & $0.53 \mathrm{x}$ & 0.08 & -0.23 \\
\hline 1608 & $0.74 \mathrm{X}$ & 0.06 & -0.14 \\
\hline 1609 & $0.59 \mathrm{x}$ & 0.39 & -0.15 \\
\hline 1611 & $0.37 \mathrm{x}$ & 0.16 & -0.29 \\
\hline 1612 & $0.47 \mathrm{X}$ & 0.07 & -0.29 \\
\hline 1614 & $0.70 \mathrm{x}$ & 0.17 & -0.07 \\
\hline 1618 & $0.58 \mathrm{x}$ & -0.18 & -0.45 \\
\hline INV07 & $0.48 \mathrm{x}$ & 0.27 & -0.23 \\
\hline INV10 & $0.71 \mathrm{x}$ & 0.12 & 0.04 \\
\hline
\end{tabular}

\begin{tabular}{|l|c|c|c|}
\hline \multicolumn{4}{|l|}{ Participantes que integran el perfil 2 } \\
\hline $\begin{array}{l}\text { Cádigo del } \\
\text { participante }\end{array}$ & $\begin{array}{c}\text { Factor } \\
\mathbf{1}\end{array}$ & $\begin{array}{c}\text { Factor } \\
\mathbf{2}\end{array}$ & $\begin{array}{c}\text { Factor } \\
\mathbf{3}\end{array}$ \\
\hline AUT02 & -0.01 & $\mathbf{0 . 5 0 X}$ & -0.24 \\
\hline AUT03 & 0.29 & $\mathbf{0 . 3 6 X}$ & -0.21 \\
\hline AUT04 & 0.25 & $0.53 \mathrm{X}$ & -0.12 \\
\hline INV02 & 0.01 & $\mathbf{0 . 4 4 X}$ & -0.36 \\
\hline INV03 & 0.11 & $\mathbf{0 . 6 1 X}$ & 0.09 \\
\hline INV04 & 0.16 & $0.59 \mathrm{X}$ & -0.05 \\
\hline INV05 & 0.39 & $\mathbf{0 . 6 0 X}$ & -0.10 \\
\hline INV08 & 0.25 & $0.50 \mathrm{X}$ & -0.31 \\
\hline INV09 & 0.18 & $0.55 \mathrm{X}$ & 0.00 \\
\hline INV11 & 0.20 & $\mathbf{0 . 4 9 X}$ & -0.42 \\
\hline INV12 & 0.03 & $\mathbf{0 . 4 3 X}$ & -0.05 \\
\hline
\end{tabular}

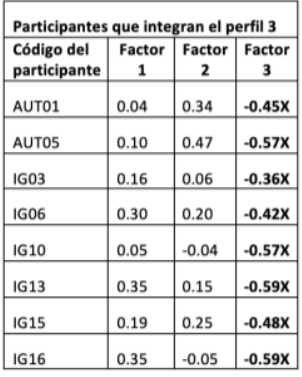

El factor 1 explica el 14\% de la varianza y esta asociada a 11 opiniones; el segundo factor explica el $12 \%$ de la varianza agrupando otras 11 opiniones; por último, el factor 3 explica el $11 \%$ de la varianza y contempla 8 opiniones.

Tabla 3. Afirmaciones con mayor nivel de acuerdo y desacuerdo para cada factor
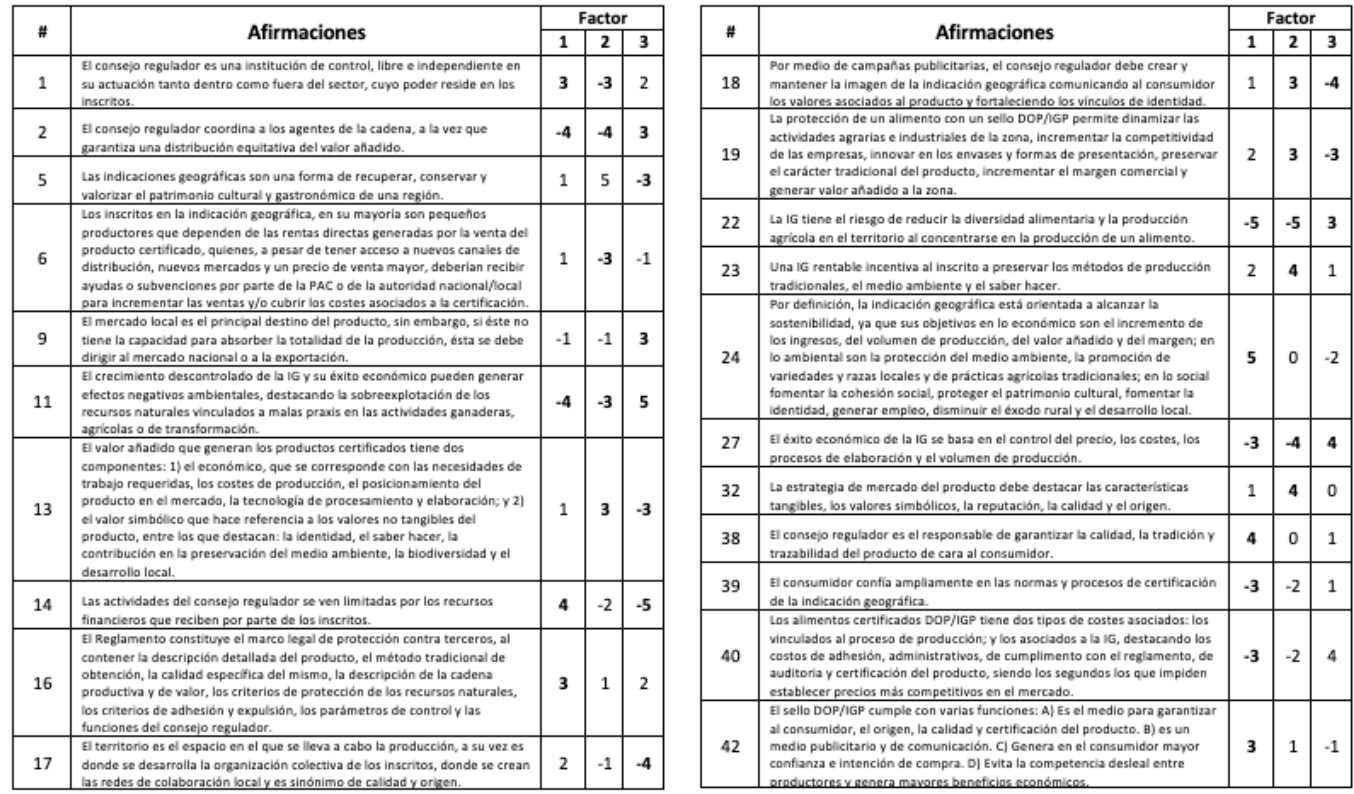

En la Tabla 3 , se presentan las afirmaciones del Q-Set que obtuvieron los valores más altos $(+5$, $+4,+3)$ y los 6 valores más bajos $(-5,-5,-3)$ en cada factor, las cuales se emplearon para estructurar el 
argumento y definirlos respectivamente como: visión institucional de gobernanza; identidad cultural y valores simbólicos intangibles; la IG como promotora de la sostenibilidad.

\subsection{Factor 1. Visión institucional y de gobernanza.}

Este argumento destaca principalmente los objetivos planteados por la Unión Europea, al momento de implementar las IG, desde una perspectiva local, destacando los sistemas de producción como factores de desarrollo local y de sostenibilidad, cuyo objetivo es preservar, proteger y promover un alimento, respetar un territorio y fomentar un saber hacer tradicional, apoyado mediante un reglamento y un pliego de condiciones como ejes de actuación de los CR. En esta visión, el CR tiene una participación administrativa, de gestión y coordinación, siendo la pieza fundamental para la gobernanza y la existencia de la IG.

Este discurso se sustenta por la opinión de responsables de IGs pequeñas, constituidas por pequeños productores, con una producción limitada orientada al mercado local. Este grupo destaca por la importancia que tiene la IG como factor de cohesión territorial, generando beneficios económicos y sociales para las pymes. Opinión que comparten las IG con un mayor potencial de mercado previenes de alimentos emblemáticos españoles, principalmente jamones y aceites, quienes destacan los valores culturales y tradicionales del producto como principal herramienta de mercado. Por ultimo se cuenta con la opinión de investigadores cuya actividad académica coincide con los alimentos mencionados, generado una asociación entre academia e IGs.

\subsection{Factor 2. Identidad cultural y valores simbólicos intangibles}

El argumento destaca los elementos característicos de la IG, la importancia de la tradición y del patrimonio gastronómico local, factores que permiten la cohesión social, generar identidad y pertenencia al territorio. Los valores intangibles del producto son los que pueden ser explotados pro las empresas inscritas para la generación de beneficios económicos, generando una dinámica que permitirá preservar los sistemas tradicionales de producción, incentivar la competitividad y la innovación de los elementos diferenciados del producto frente a los productos no certificados. En este argumento se destaca que el crecimiento de una IG genera desarrollo local y no conlleva efectos negativos sobre el medio ambiente.

Este argumento se construye con la opinión del colectivo académico, cuyos trabajos han destaco los elementos diferenciados de los alimentos IG, destacando los beneficios económicos, en especial el potencial de crecimiento del mercado y la generación de mayores ingresos. Igualmente se cuenta con la opinión de representantes de los gobiernos autonómicos que cuentan con un alto numero de productos DOP/IGP.

\subsection{Factor 3. La IG como promotora de sostenibilidad}

$\mathrm{El}$ argumento parte de una visión institucional asociada a la imagen de la marca, incluyendo una mayor preocupación por la sostenibilidad de los sistemas de producción al considerar el riesgo de efectos negativos que se pueden generan por un crecimiento descontrolado de la IG. Resalta la importancia de preservar los recursos naturales y de mantener un crecimiento económico controlado considerando el potencial de mercado del producto. Sitúa como organismo responsable de controlar y ejecutar las políticas internas al CR, a la vez de ser quienes debes dirigir la estrategia de mercado y vigilar el crecimiento de la IG.

El argumento se construye en torno a la opinión de $8 \mathrm{CR}$, cuyos productos están orientados a mercados locales y en el sector HORECA, la exportación no constituye un interés primordial, por el contrario, buscan una consolidación de mercado que les permita la diversificación productiva. A este grupo se suma la opinión de autoridades autonómicas que cuentan con un número reducido de IG, quienes pugnan por un sistema que valore los productos locales en lugar de una masificación de IG que ponga en riesgo al medio ambiente y al propio producto IG. 


\subsection{Análisis de los resultados}

Los perfiles obtenidos han permitido identificar las motivaciones que subyacen en la implementación de las IG en España, además de conocer las principales características de los participantes que las sustentan, a partir de esta información, es posible determinar algunos puntos de actuación para modificar la manera en que se aplica y perciben las IG.

El sistema IG cuenta con un gran numero de beneficios que las hace atractivas tanto a productores como a consumidores, sin embargo, no todos los objetivos planteados desde la Unión Europea son compartidos por las diferentes DOP/IGP. Por una parte, este sistema se aplica a productos tradicionales con una alta reputación y valores culturales, la forma en que se establecen omite algunos objetivos, en especial medio ambientales y sociales, disminuyendo con ello el alcance que podrían tener en el territorio.

\section{Conclusiones}

Desde la perspectiva de los actores involucrados en las IG, estas se perciben como un sistema de certificación que crea beneficios, notablemente económicos, a los inscritos. Adicionalmente representa una herramienta que permite el desarrollo local, la preservación del patrimonio gastronómico local o la sostenibilidad. Sin embargo, es necesario crear estrategias que permitan que las tres perspectivas subsistan y se complementen, ya que constituyen parte de los objetivos europeos y se ha demostrado que una correcta aplicación de las IG genera beneficios en lo económico, lo medio ambiental y lo social, por lo cual pueden ser consideradas como herramientas que permitan consolidar un sistema agroalimentario sostenible en España.

\section{Bibliografía}

Brown, S. R. (1993). A Primer on Q Methodology. Operant Subjectivity, 16(3/4), 91-138.

Darton, R. A. (1980). Rotation in Factor Analysis. Journal of the Royal Staistical Society, 29(3), $167-194$.

Esnouf, C., Russel, M., \& Bricas, N. (Eds.). (2013). FOOD SYSTEM SUSTAINABILITY Insights from duALIne. Cambridge University Press. https://doi.org/10.1176/pn.39.24.00390022b

Fierro, J. C., \& Martín, A. V. (2009). Denominaciones de Origen e Indicaciones Geográficas: justificación de su empleo y valoración de su situación actual en España. Mediterráneo Económico, 15, 329-350.

Frutos Mejías, L. M., \& Ruiz Budría, E. (Eds.). (2012). Los productos con indicación geográfica en el sistema agroalimentario español. Tradición y modernidad. https://ifc.dpz.es/recursos/publicaciones/32/28/_ebook.pdf

García Romana, A. (2017). “DENOMINACIONES DE ORIGEN E IMPACTO EN EL DESARROLLO RURAL EN EXTREMADURA” [Universidad de Extremadura].

http://dehesa.unex.es/bitstream/handle/10662/5549/TDUEX_2017_Garcia_Romana.pdf?sequence=1\&isAllowed=y

Millán Vázquez De La Torre, G., Morales Fernández, E., \& Pérez Naranjo, L. M. (2014). Turismo gastronómico, Denominaciones de Origen y desarrollo rural en Andalucía: situación actual. Boletín de La Asociación de Geógrafos Españoles, 65, 113-138. https://doi.org/10.21138/bage.1746

Ramírez García, S. (2016). Desarrollo rural y calidad diferenciada. Análisis de las dinámicas de las denominaciones de origen en la Comunidad de Madrid [Universidad Complutense de Madrid]. https://eprints.ucm.es/39201/1/T37811.pdf

Ramlo, S. (2016). Centroid and Theoretical Rotation: Justification for Their Use in Q Methodology Research. Mid-Western Educational Researcher, 28(1), 73-92.

Roth Deubel, A.-N., \& Bernal Gamboa, E. (2014). Metodología Q: una alternativa para la participación en la reforma de la política de ecudación superior en Colombia. Ciencia Politica, 9(18), 237-264. 